\title{
Influence of COVID-19 Pandemic in Students' Distribution of Time in Distant Learning
}

P. Rodriguez ${ }^{1}$, S. Atrio ${ }^{2}$ and G. M. Sacha ${ }^{1 *}$

1. Departamento de Ingeniería Informática. Escuela Politécnica Superior.

2. Departamento de Didácticas Específicas, Facultad de Formación de Profesorado y Educación.

Universidad Autónoma de Madrid.

Ciudad Universitaria de Cantoblanco, 28049, Madrid, Spain.

* sacha.gomez@uam.es

Short Title: Distant Learning Time Distribution in COVID-19 Pandemic 


\section{Abstract}

Statistical analysis offers unprecedented opportunities to identify learning strategies. This fact has been boosted in the COVID-19 pandemic because of the data obtained in distant learning scenarios due to the confinement. This article deals with the identification of students' strategies in different distant learning scenarios such as working autonomously as a support for face-to-face classes or learning autonomously in COVID-19 confinement. We have measured the influence of parameters such as the time they spent in self-evaluation, the scores obtained through this process and the distribution of time when studying autonomously. We have only detected significant results that guide to a better learning strategy when we include time parameters, such as the time between studying sessions or the time between students' first session and their final exam. We demonstrate that students that started to study earlier and more dispersed get better success ratio (not necessarily better scores) than those that started later and do it more concentrate. The findings from this study suggest that the same amount of time spent in autonomous learning optimizes its effectiveness when it is extended in time. This learning strategy was found more often in COVID-19 confinement, where students were forced to stay at home.

Keywords:

e-Learning

Computer Adaptive Testing

COVID-19

Higher Education 


\section{Introduction}

COVID-19 pandemic has influenced in almost all aspects of our lives. Higher education is not an exception (Aristovnik, Ravšelj, \& Umek, A Bibliometric Analysis of COVID-19 across Science and Social Science Research Landscape, 2020). Because of the confinement applied in most of the countries, learning and teaching strategies has changed dramatically [1], being the most important difference the more intensive application of distant learning methods. In this new scenario, students have changed their learning habits and their academic results have been also altered. Time management is one of the most relevant parameters when explaining the effects of COVID-19 confinement in students' performance. Moreover, the use of computers in education (e-Learning) have been a key tool for doing a complete and quantitative analysis.

E-Learning can be defined as the methods, tools and resources that apply communication and information technology in education. The progress of these technologies has made e-Learning a very effective tool in COVID-19 confinement [2]. It is clear that, if this situation would happen years before, the difficulties to overcome teaching in confinement would have been much bigger.

In the present article, we analyze the effect of time distribution when studying autonomously. By doing so, we start showing a study performed before the pandemic and its results. Then, we analyze the effect of time distribution when studying in the COVID-19 pandemic and compare the results with the previous findings. This is a very important part of the article because it gives us a vision of the particular situation lived by the students in the pandemic, which can be indeed very different than previous scenarios where they also faced distant learning (such as MOOCS, for example).

For data acquisition, we propose the implementation of a Computer Adaptive Testing (CAT) technique that can be used both as self-evaluation and assessment tool to analyze students' learning strategies and their efficiency as a function of time distribution. As we will demonstrate, by using this technique and all the statistical information extracted through the web application where they were implemented, we can extract some of the most important parameters of autonomous learning such as the time spent when using the application, their scores and the exact dates and hours of autonomous learning activities. In previous works [3], it has been shown that most of the students tend to learn just the correct answers instead of the subject contents involved in the questions. In this article, we demonstrate that the amount of time spent in autonomous learning correlates with final scores only when the distribution of time is uniformly distributed throughout the learning process. Students who spent most of the time in the last few days before the evaluation process can be also succeeded, but the probabilities of failure strongly increase. These conclusions will be compared with the results obtained in the COVID-19 pandemic, where we got data from different scenarios (pre-pandemic, full confinement and pos-confinement).

\subsection{Literature review}


Several studies have demonstrated that, in some cases, students' performance increased in COVID-19 confinement and several explanations have been reported [1,4]. In other cases, negative effects have been reported, such as the lack of feedback by the teachers [5], limited access to technology [6] or wrong teachers and students' behavior [7]. It has been also reported that students were psychologically affected by the pandemic and, sometimes, their perception about their own work were not realistic compared to their real performance [8]. For this reason, it is very important to separate students' beliefs when analyzing efficiency in learning. Of course, emotional impact in students can be one of the most important facts in their own performance [9], but still they usually felt that they were doing worse due to their negative feelings in the pandemic. It is also worth noting that different opinions can be found from students and teachers. For example, it has been reported that students appreciate much more than teachers the efficiency of online lectures [10]. In general, negative and positive effects were always reported from same groups. For example, low-income families had more problems by accessing to technology, but at the same time, students from these families were also found to feel more belonging to the learning community [11].

However, as it happens with any emerging technology, the progress also implies some disadvantages that must be considered for teachers when facing the development of a new course, subject or activity. For example, it is known previously to COVID-19 that the large number of potential students that can access to courses such as Massive Open Online Courses (MOOCs) [12] creates new challenges in activities such as monitoring the learning processes. Obviously, in distant learning forced by COVID-19 confinement, this scenario also applies. For these reasons, the use of information technologies in learning must be always accompanied by a quantitative and accurate data analysis. [13] Fortunately, many advantages can be also found, highlighting a higher elasticity in when and how learn compared to traditional strategies, which is one of the most important concepts to take into account in confinement. The main learning activities in this new paradigm are related to autonomous learning, which is a very difficult concept when measuring quantitatively [14].

Other studies have found that variables that influence the completion rate are the level of education [15] and the time management $[16,17]$, which is one of the topics of the present article. When looking for models that help in the prediction of academic success, one of the best parameters is the time spent using the computer-based tutoring system [15].

Since the main objective of this article is to analyze and identify the most efficient autonomous learning strategies and their time distribution, we must focus on the best way of measuring students' activities when working autonomously. In the past, self-reported surveys have been commonly used for data collection when investigating autonomous learning [18]. However, it contains errors such as having students as the main data source since they are directly involved in the learning process and are subjective because of their own involvement in the learning activities. In this scenario, developing learning activities that can be easily monitored and give accurate information about students' performance is necessary. A very good candidate for this task is CAT because of their ability to adapt the content presented to learners as a function of their responses [19]. CAT has been commonly used in a wide variety of courses and activities: language [20], identification of learning styles [21,22], 
mathematics [23], cognitive diagnostic assessment [24] and programming [25]. However, the advantages of using CATs in learning implies also a deep knowledge of the numerical models underneath to make a good calibration of the system [26,27].

\section{Materials and methods}

\subsection{Measurement instruments}

We have used the e-valUAM software as the platform for obtaining data both for self-evaluation and final exams. E-valUAM has been described previously [4], and only a brief description is given here. The main idea of this application is to apply an assessment process where students are evaluated through adaptive tests as described in following paragraphs. This method allows us to order contents by importance, relevance or difficulty. The basic idea is that test items are selected by the computer to individually match users' ability level.

The idea of this model is that all students will need to understand firstly the contents that teachers consider more relevant (included in the first levels).

In the example shown in figure 1, Newton's second law is considered basic knowledge that must be placed as a level 1 question. Complex problems involving several forces and gravity would be included as level 3 questions. By doing so, teachers avoid the usual behavior of some students that only take care of the complex problems frequently asked in an exam, without taking care of the basic knowledge underneath. In other words, complex problems are only asked when students have demonstrated a robust basic knowledge of the subject.

Fig. 1: Left: Main numerical model of the CAT system included in e-valUAM. Right: Examples of items that belong to the first (upper) and last (lower) level.

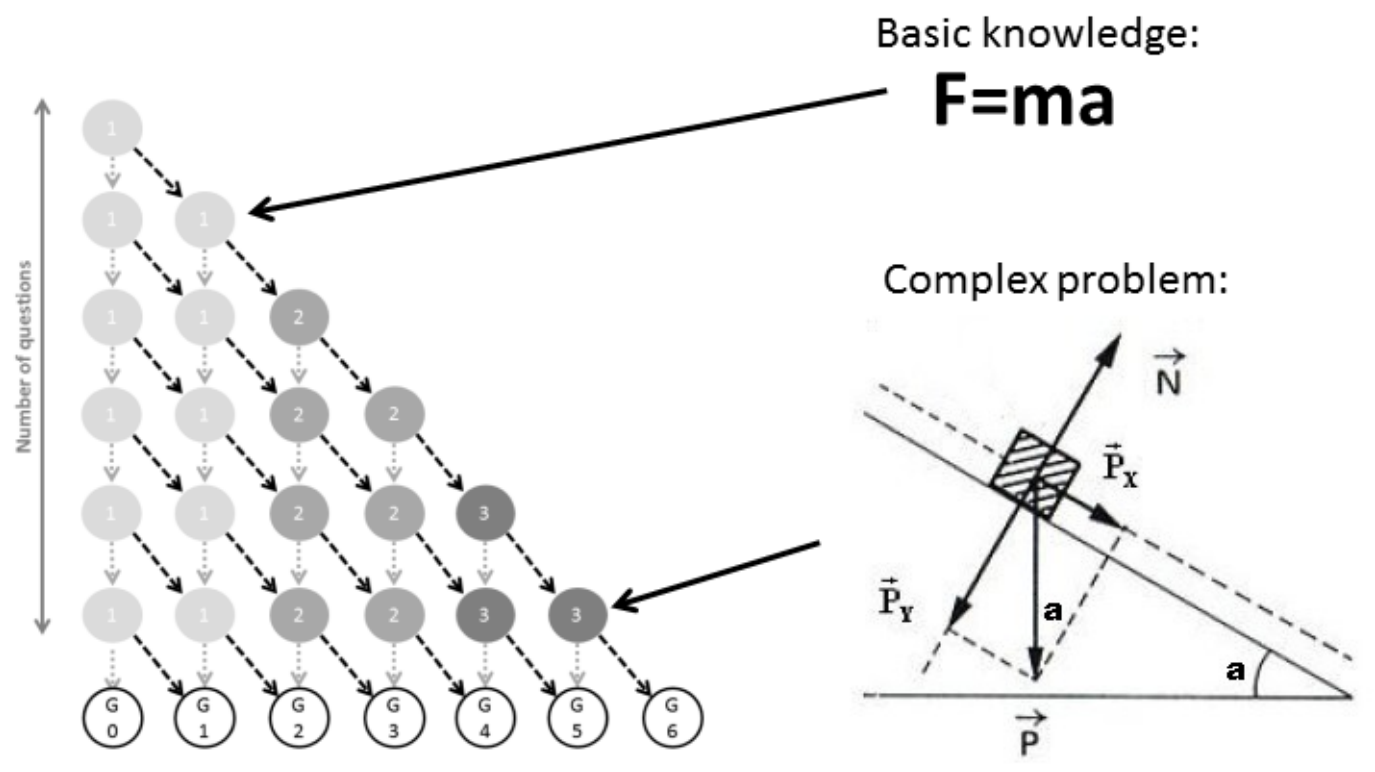




\subsection{Design of the experiments}

\subsubsection{Influence of time management in students' performance}

In this first study, we are analyzing the effect of different autonomous learning strategies, focusing on time management. For this first study, we have used Multiple Answer tests (MA-T), which are the most suitable format in the subject under study (Early Childhood Education course). We have used a standard format where a single correct answer must be chosen from a pool of possible answers shown by the system.

The adaptive test developed for the final exam was divided in 4 levels (10 questions per level). By applying these parameters to the model shown in figure 1 , we obtain that students had to answer 40 questions. More details about the questions and levels included in the test can be found in the literature [22].

In figure 2 we show two questions extracted from the test described before, that was used both for autonomous learning and final exam. It is worth noting that questions in the final exam were different in the sense that some data were changed. However, the amount and nature of questions did not change from the autonomous learning phase. In the figure we show an easy question related to the components of air, which corresponds to level 1 , and could be asked any time a question from this level is shown. Questions are selected randomly from a repository, so it is impossible to know exactly when a question will appear. The only condition that is mandatory is to get a question from the repository of the level that corresponds. It is also shown in the figure a more complex question, included in the level 3 repository. In this case, the student should answer the technical name of a plant by identifying it by a picture. We can also observe in figure 2 the two possible feedbacks given by the e-valUAM interface (wrong and right previous questions respectively). It is worth noting that these questions were extracted for the autonomous learning phase since teachers decided not to give any feedback in the final exam. The use of this system implies a huge engagement of students because of two reasons.

1) The adaptive test reduces student's frustration when facing very difficult questions since these questions are only asked when the student starts having correct answers from previous and easier questions.

2) By using the same format in the autonomous learning and final exams, students are tempted to use the platform since it is a very accurate training activity.

Those characteristics of our methodology give us the opportunity of analyzing accurately the autonomous work of students by measuring their activity when connected to the platform. 
Fig. 2. E-valUAM interface for the test used in autonomous learning and final exam

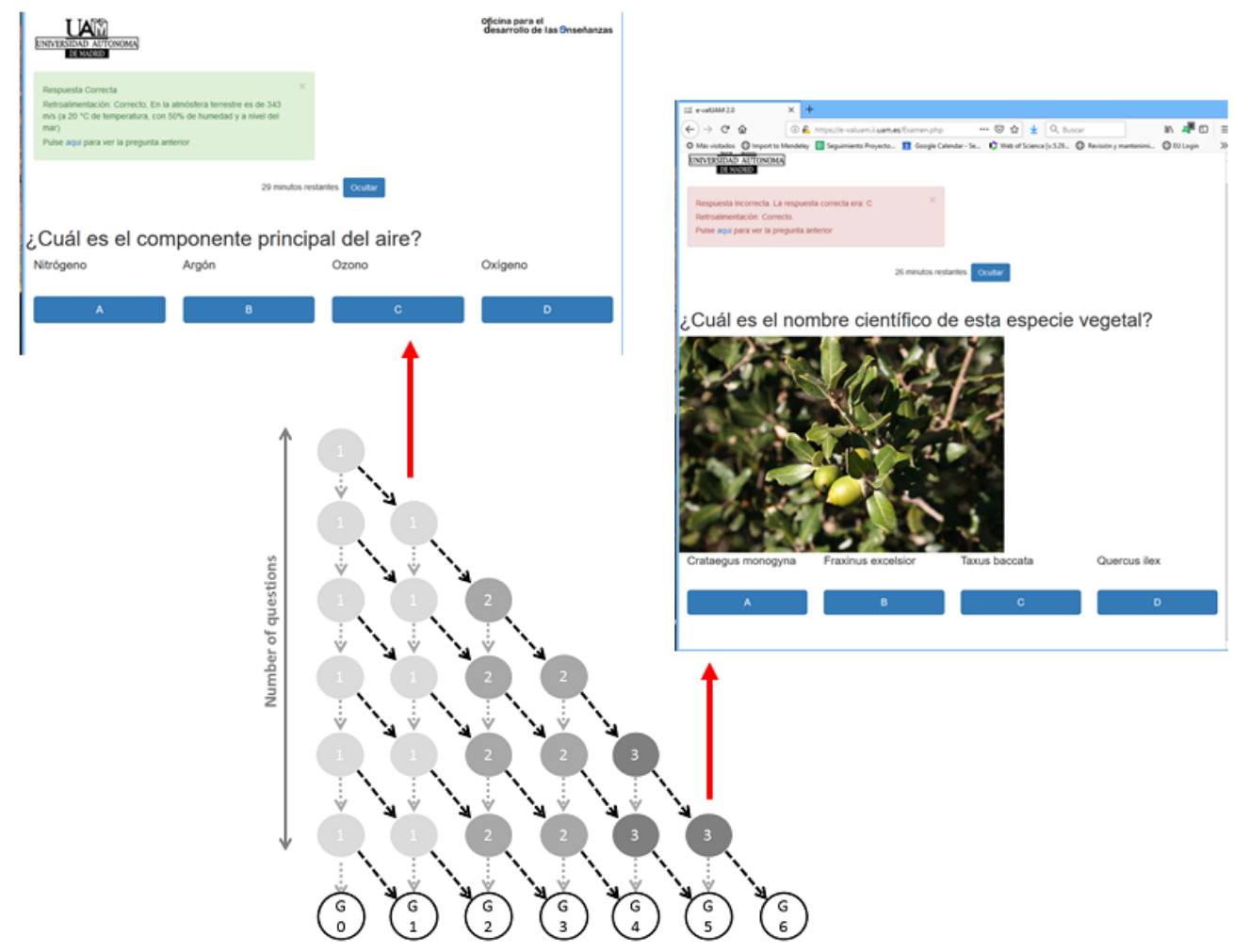

\subsubsection{COVID-19 confinement influence in students' management of time}

In this part, our goal is checking the influence of continuous working extending the analysis to a study that involves 5 sets of students from a subject that did not change its conditions in five years. This subject had the same contents, teachers, teaching methods, exams and online material in the whole five academic courses. What makes it interesting is that, due to the COVID-19 confinement, we can include data from 3 scenarios that only change the face-to-face or distant learning.

1) Pre-COVID19. In this first scenario, both teaching and assessment were done face-to-face. It happened in academic years 2016/2017, 2017/2018 and 2018/2019.

2) COVID-19 confinement. This happened in academic year 2019/2020.

3) Post-COVID-19 confinement. During academic year 2020/2021, students were not confined at their homes, so some restrictions could be softened. In the case of our subject, teaching was online but final exams were face-toface.

\subsection{Participants}

The experiment, that includes two different stages that will be described as described before, has been performed with students from two different degrees. 
The first part has been performed with a set of 44 students from the fourth course of the Degree in Early Childhood Education at the Universidad Autónoma de Madrid.

The second part has been performed in five academic courses (from 2016/2017 to 2020/2021) with a set of 110 , 78 , 79, 86 and 73 students each year in the first-year subject "Applied Computing" of the Degree in Chemical Engineering at the Universidad Autónoma de Madrid. This subject was taught through theory lessons and practical classes in the computer laboratory. This course corresponds to 6 ECTS. Due to COVID-19 pandemic, the face-to-face teaching was cancelled from March 11 to mid-May (end of semester) in 2019/2020 course. In 2020/2021, all classes were taught online but final exams were face-to-face.

\section{Results}

In previous works, it has been shown [22] that the relation between the scores obtained by the students along the selfevaluation process and the scores achieved in the final exam do not always have a clear relationship about the learning strategy in terms of efficiency at the final exam. In this section we propose to analyze their learning strategy taking also into account the dynamics involved in their learning process, i.e. including time as a new relevant parameter.

Fig. 3: Temporal distribution of students' accesses. Temporal line starts when the application is available to the students for the first time and finishes at the time when the final exam is done. Students are identified by an anonymous identification number (Id).

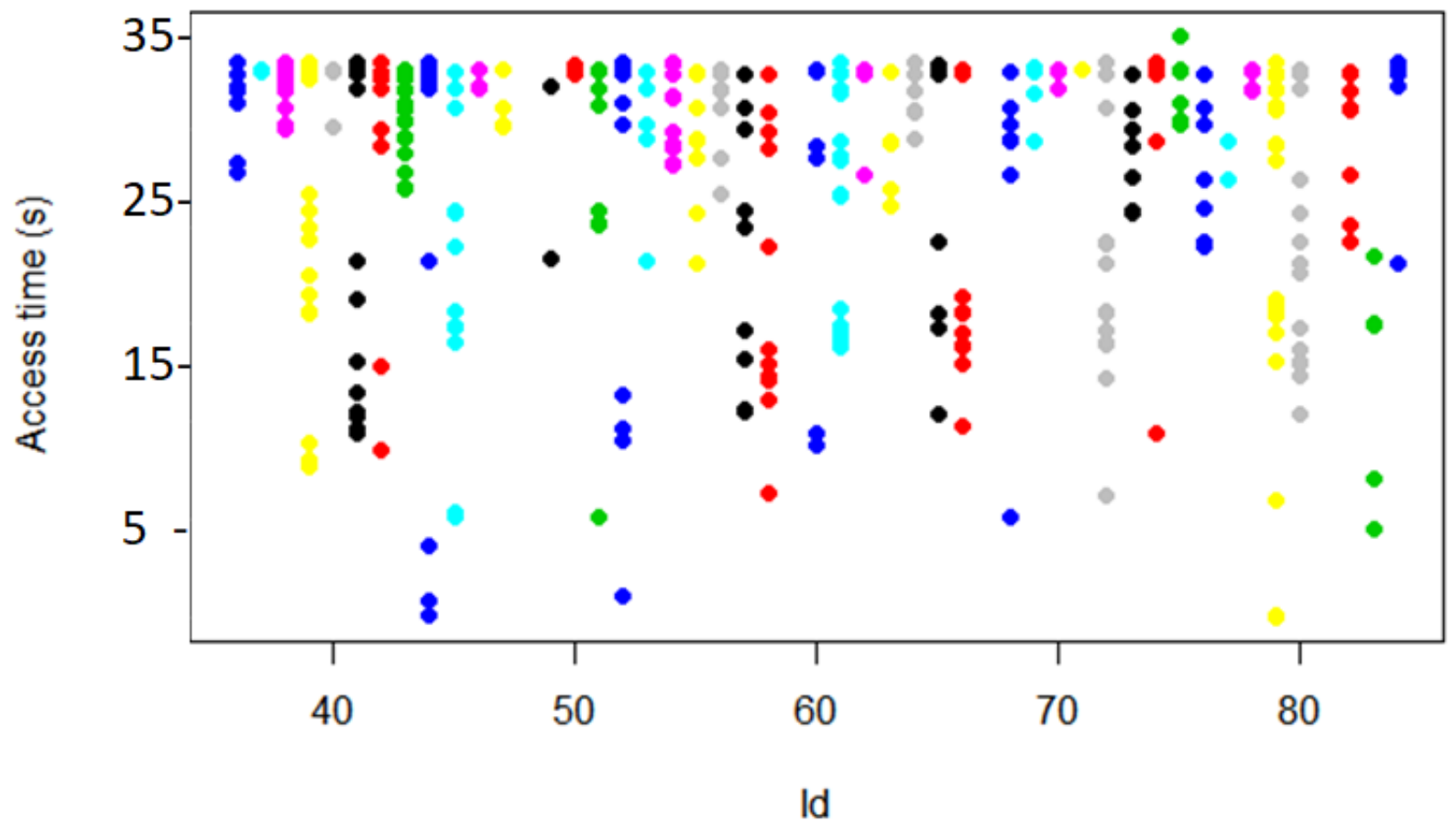


In figure 3 we can see the times the students used the application through the self-evaluation process. We can see in the figure a clear tendency to the upper limit, which is the time when the final test was done. This represents the common scenario when students make their best effort only when the final exam is approaching. We can see even that some students only used the application one or two days before the exam. On the other hand, we can also observe that some of them used it from the beginning. Clearly, these different behaviors can lead students to different degrees of success in their assessment process. For this reason, we must go further in the analysis by defining a variable related to the time distribution and comparing it with the scores from the final exam. In figure 4 we compare the number of correct answers in the final exam with the mean access (MA) of all the students, defining MA as the mean value of the date obtained by including each time a student has been using the application. As we can see in figure 3, the origin of time corresponds to the very first moment when the application was available to the students. In figure 4 we find that all MAs are included in a window between days 18 and 33 (final exam was done at day 33).

Fig. 4: Mean Access (MA) as a function of the number of correct answers for all the students included in the study. Students are identified by an anonymous identification number.

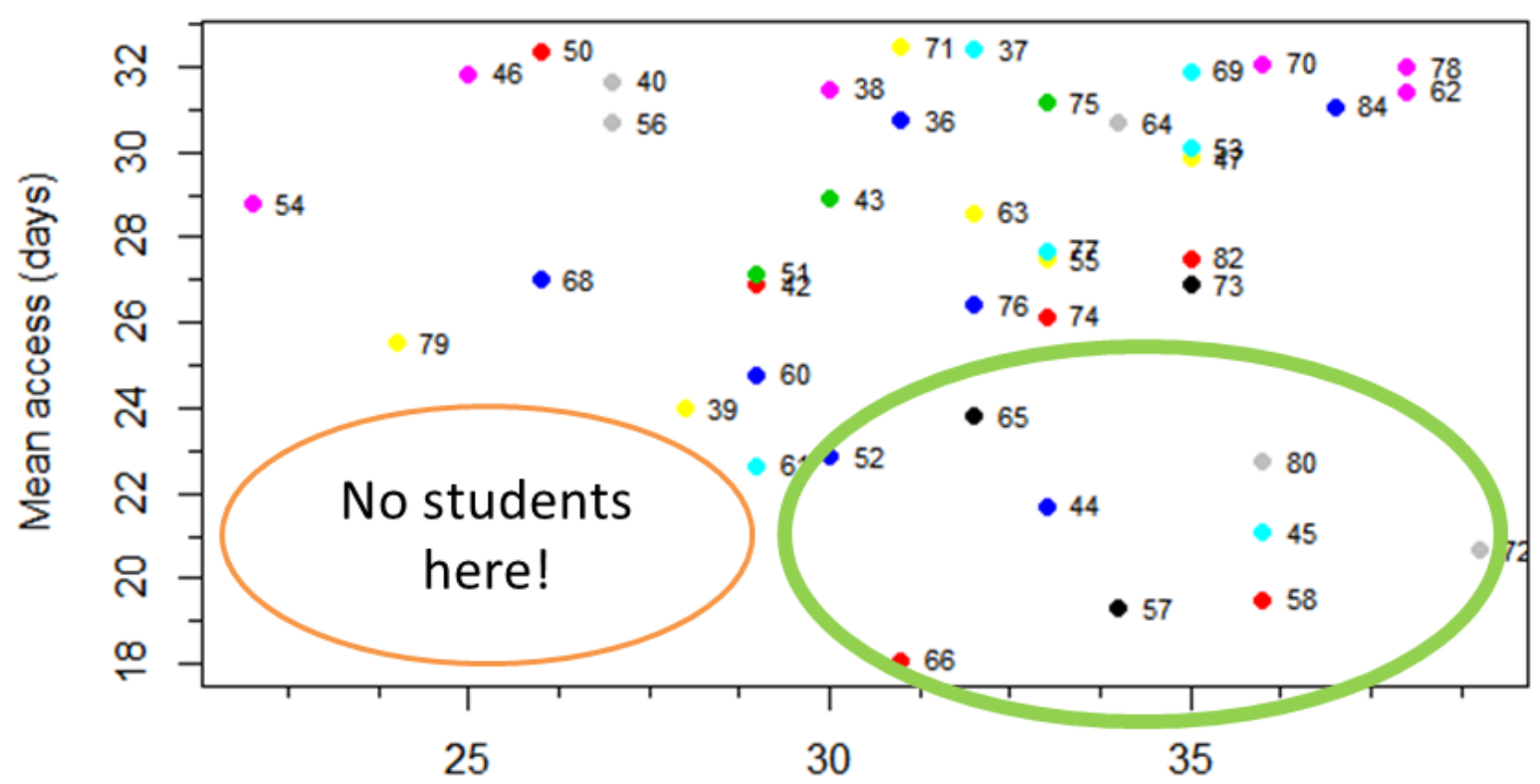

Correct answers

As we can see in figure 4, there is a region where no single student is placed. This region (circled in the figure and labeled "no students here!") corresponds to low MA values and low scores. The absence of students in this area suggests that using the application from the beginning is a good strategy to get good scores. This fact, however, does not imply that using a last-minute strategy is necessary bad since, in the upper side of the figure, we can find students with both low and high scores. In other words, a strategy that implies a continuous use of the application guarantees the success, which is a well-stablished paradigm. Most shocking result is that a last-minute strategy does not necessarily imply failure. Actually, more than $50 \%$ of 
students obtained good scores in the final exam with a strategy of using the application the very last few days. By following those results, we cannot say that studying with a distribution of time that focuses all the efforts in a few days leads necessary to failure. We can only say that a more homogeneous distribution of time is a strategy that reduces the chances of failure.

However, these results are not enough if we want to analyze the whole temporal line from figure 5 . If we focus, for example, on students 58 and 66, we can see that they show similar MA values and, however, their temporal distribution is pretty different. Student \#58 shows a much more homogeneous distribution in figure 5 than student \#66, who used the application several times between days 12 and 20 and did not use the application again until the last two days. This behavior is clearly different and ended in different scores, with a much better perform for student \#58.

To take into account the variability of the temporal distribution, we have defined the Mean Time Between Accesses (MTBA) as the mean value of the elapsed time between each access and the next one. We have considered that students with a high MTBA (students that study separating more the accesses) should be students with good performance. In figure 5 we represent the MTBA identifying the students with low MA in figure 6.

Fig. 5: Mean time between accesses for all the students included in the study. Students are identified by an anonymous identification number.

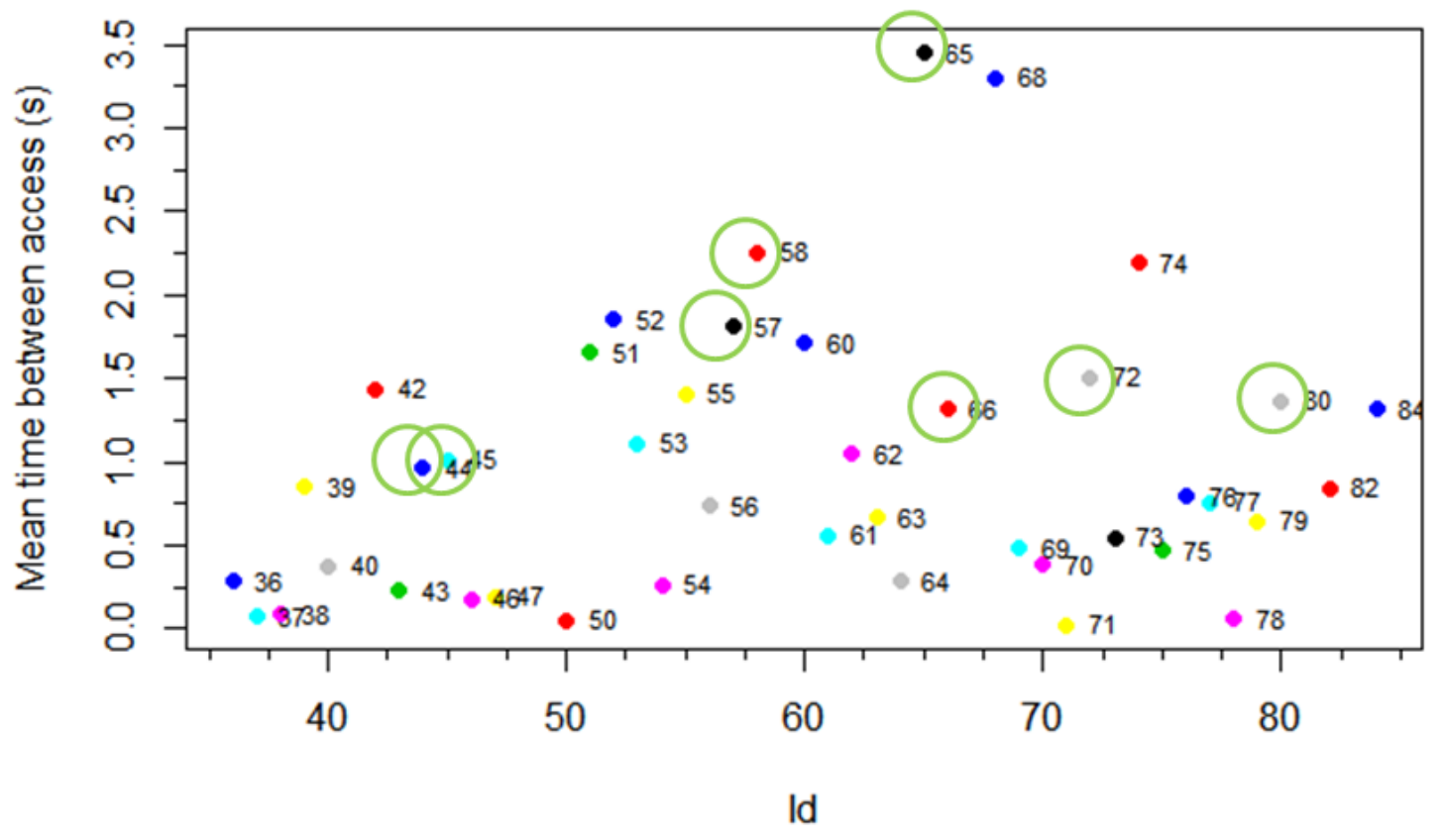

To verify this hypothesis, we have performed Welch's t-tests. We have considered two different groups: The students circled in green in figures 4 and 5 with low MA and high MTBA (group 1) and the rest of students with a high MA and low MTBA (group 2). In other words, the hypothesis is that group 1 get higher grades than group 2.

In all the tests, we compare MA and MTBA and, if there was a significant difference between both, we then look for a significant difference in the grades. We started with groups of six students with the lowest MA from group 1 and we tested for a 
lower mean MA and bigger MTBA from group 2 grades. Being significant in the three variables, we add a new group 1 member. If the new member keeps the difference between groups significant, we add a new one and repeat the tests. If not, we move back that new student to group 2 and repeat the operation with a new student. Applying the Welch's t-test, we obtained the following results, (being $\alpha=.05)$ :

Table 1. Welch's t-test results.

\begin{tabular}{|c|c|c|c|c|}
\hline \multicolumn{2}{|l|}{ Group tested } & MA & MTBA & Grade \\
\hline \multirow[t]{2}{*}{ G0 } & $\mathrm{t}$ & -11.172 & 2.434 & 2.8267 \\
\hline & p-value & $3.659 \mathrm{e}-09$ & 0.03333 & 0.02028 \\
\hline \multirow[t]{2}{*}{$\mathrm{G} 0+80$} & $\mathrm{t}$ & -10.471 & 2.6631 & 3.4134 \\
\hline & p-value & $1.031 \mathrm{e}-08$ & 0.01672 & 0.004939 \\
\hline \multirow[t]{2}{*}{$\mathrm{G} 0+80,78$} & $\mathrm{t}$ & -4.139 & 1.4655 & \multirow{2}{*}{$\begin{array}{l}\text { Not tested (as } \\
\text { MTBA is not } \\
\text { significant) }\end{array}$} \\
\hline & p-value & 0.002735 & 0.1654 & \\
\hline \multirow[t]{2}{*}{$\mathrm{G} 0+80,76$} & $\mathrm{t}$ & -7.2182 & 2.259 & 3.2048 \\
\hline & p-value & $9.808 \mathrm{e}-06$ & 0.03537 & 0.005622 \\
\hline$\cdots$ & $\cdots$ & $\cdots$ & $\cdots$ & $\cdots$ \\
\hline \multirow[t]{2}{*}{$\mathrm{G} 0+80,76,74,65,73$} & $\mathrm{t}$ & -6.2413 & 2.9014 & 3.4844 \\
\hline & p-value & $8.288 \mathrm{e}-06$ & 0.01043 & 0.001513 \\
\hline
\end{tabular}

As we can see in table 1, students with low MA and high MTBA (group 1) get better scores than students with high MA and low MTBA (group 2).

After stablishing the importance of time management, we will analyze now the effect of COVID-19 confinement in students' learning strategies, paying attention to the time distribution as we did in previous section. In confinement year (2019/2020), students only could stay at home. In previous years and post-COVID-19 confinement, they were able to do many activities outside their houses and (with some restrictions in 2020/2021) meet with friends or family. This fact, combined with the inclusion of new online teaching strategies, implied that students tended to work more continuously in COVID-19 confinement. In figure 6 , we show the number of attempts made by all the students in the five academic years analyzed. Data are divided in months. Since the subject was tough from February to May, we have included only 4 measurements in the figure. As we can see, students made a bigger effort in the very last minute in all years excepting 2020/2021. In this year, students were using the application in a much more continuous way. They even used it less in May than in April and March. This behavior is very rare, and it only happened in COVID-19 confinement, as we can see in the figure. 
Figure 6: Attempts using the application by all students of every academic year normalized to the number of students enrolled. Data are divided in months starting at the beginning of the semester and finishing at the date where the final exam is scheduled. In all cases, final exam was at the end of May.

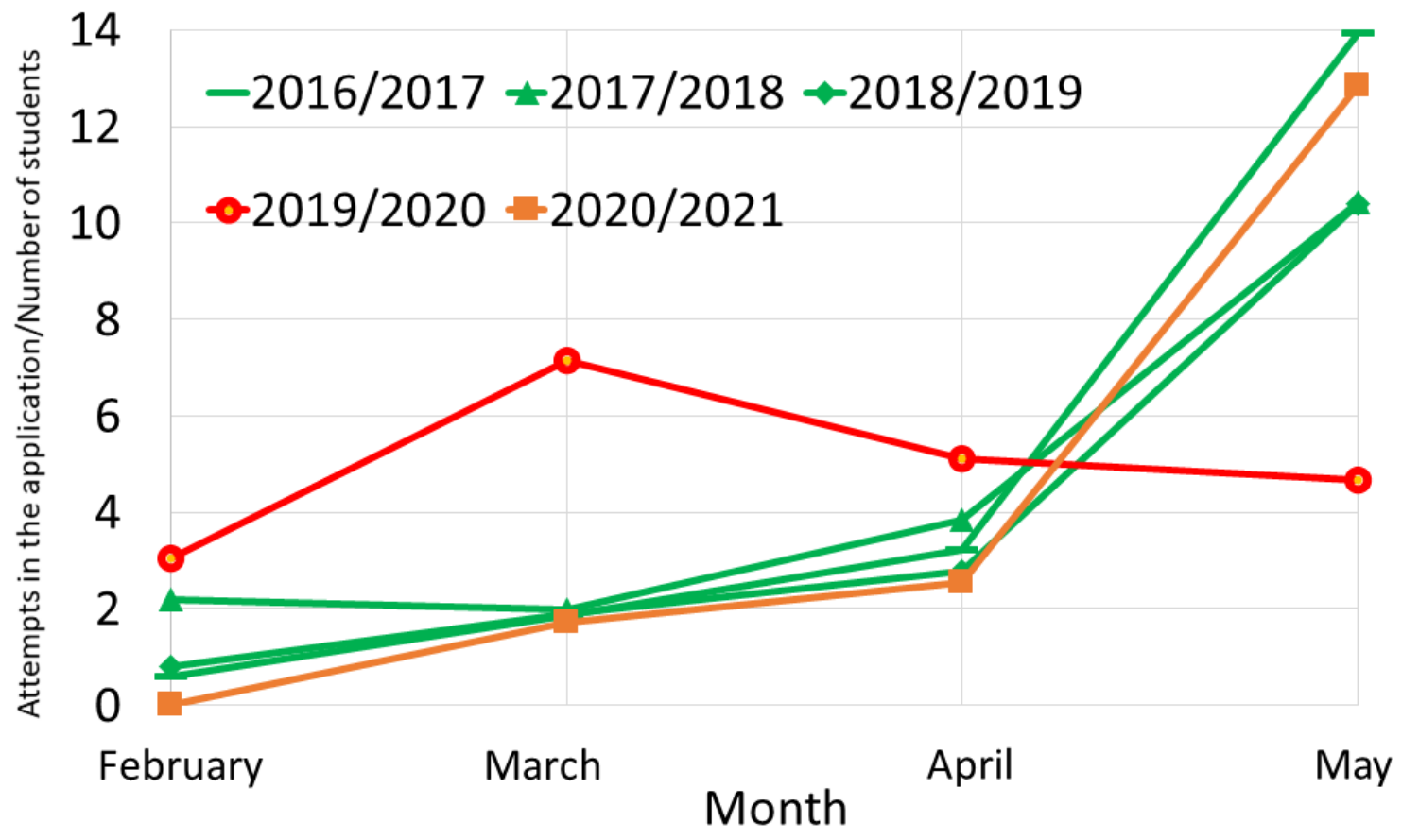

The first step to compare scores from different courses is checking the normality of our data sets from all courses. Our data sets did not pass the normality tests, which is something expected taking into account the origin of the data. We have used adaptive test with questions ordered in different levels. The test used in this case included 20 questions and 4 levels, which implies a jump every 5 questions. In other words, scores between 0 and 2.5 only includes questions from level 1 ; scores between 3 and 5 included questions from level 1 and 2; scores between 5.5 and 7.5 includes questions from levels 1,2 and 3 , and, finally, scores over 7.5 includes questions from all levels. Since questions from a higher level tend to be more difficult, a significant number of students were stacked in the scores where level increased $(2.5,5$ and 7.5$)$. This fact implied that the data did not followed a normal distribution. For this reason, all data analyzed in this section have been compared taking this fact into account.

Applying the Mann-Whitney test to the data sets, we have found that data from courses 2019/2020 and 2020/2021 and statistically different when compared to a data set that contains all scores from all the courses before the pandemic (2016/2017, $2017 / 2018$ and 2018/2019). We did not found differences between courses 2019/2020 and 2020/2021. Based on these findings, we could conclude that students' performance was higher in these two courses. However, we must be also sure that there were not statistical differences between courses before the pandemic as well. In this case, we have found that there are differences when analyzing course $2017 / 2018$ individually. We have found differences between this course and the two other courses before 
pandemic. This fact implies that we cannot assure that there were differences in the final scores before and after the pandemic because we can also find differences between different courses before the pandemic. In other words, differences in the final scores cannot be linked to the effect of the new learning strategies used because of COVID-19 confinement.

However, in agreement with the results obtained in the previous section and also in (González Gallego, et al., 2020), the number of students who quit the subject or did not pass the final exam is much lower in 2019/2020 (see figure 7) Actually, there is not a single student that failed in the final exam that year. This also happens in the following academic year (2020/2021). Actually, these two academic years, both after COVID-19 pandemic but in different confinement scenarios (more restrictive in 2019/2020), had the best results in the three parameters analyzed here (scores, fails and skip the subject). The students that skipped the subject (i.e. did not show up in the final exams) are the lowest in 2019/2020 and all of those students left the subject (which is something that happened often that year for different reasons, mainly related to the pandemic), so not a single student failed in 2019/2020.

Figure 7. Percentage of students who failed in the final exam (scored less than 5 in a $0-10$ scale) and students who did not appear in the final exam (skip) for all academic years under study.

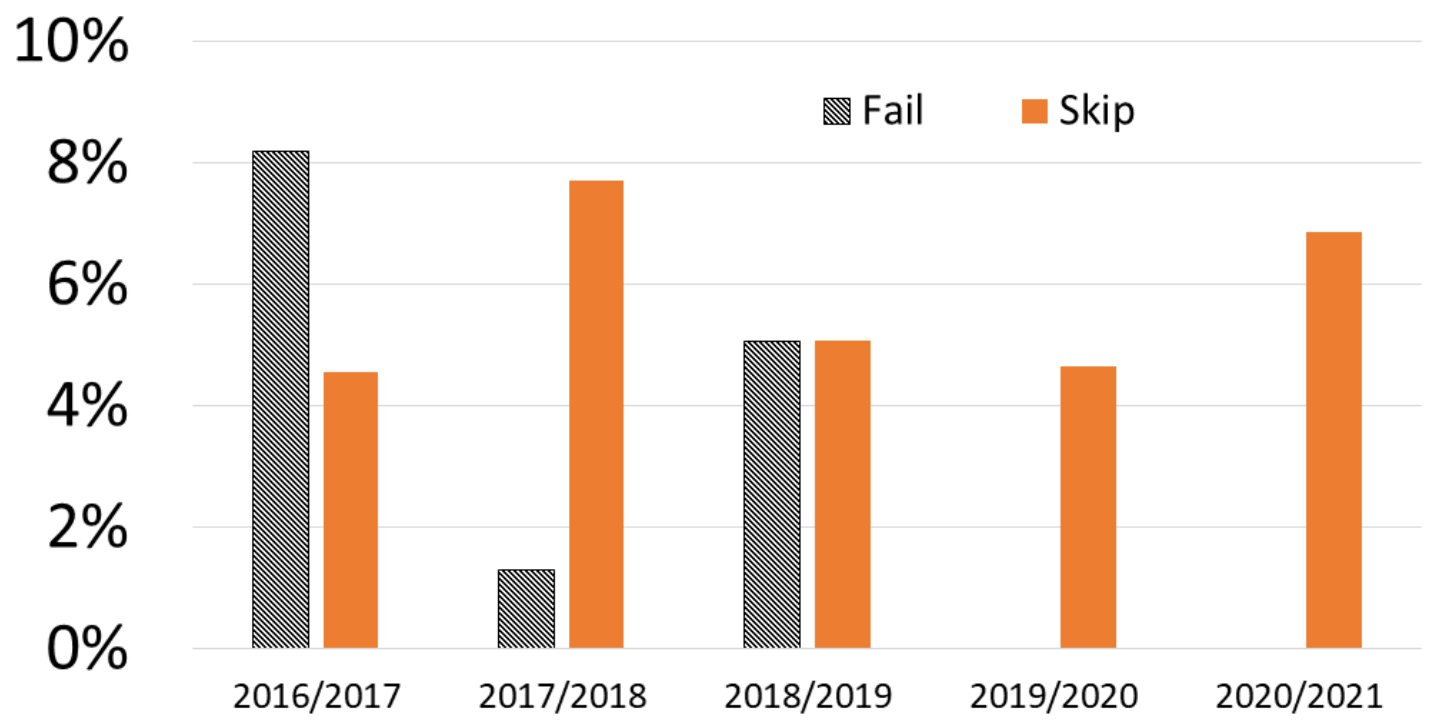

\section{Discussion}

The study done before COVID-19 confinement has demonstrated that working in a continuous basis gives a better probability of passing the course. However, working in the last minute is also a strategy that has been demonstrated to give good results. Maybe, this is the reason why this strategy is still being used extensively. Students that do not have the opportunity (or willing) to study from the very beginning of the course still have opportunities for obtaining good scores in their final exams. In conclusion, studying in a continuous basis is not the only way for getting good scores, but it is the strategy that gives the best chances of success. By doing so, it is expected that students obtain higher scores, without forgetting that an opposite strategy 
(using the application only right before the final exam) does not imply failure (as we can see in figure 4). The difference is that, in this case, lower scores also appear, which does not happen when using the group 1 learning strategy.

These findings lead us to a very important conclusion: teachers should use additional motivation strategies if they want students to work in a continuous basis. Expecting that students will see by themselves that studying in the very last minute is a bad strategy will not work always. Actually, it could have the opposite effect since students could see that it is actually a strategy that gives good results.

COVID-19 pandemic created a brand-new situation where students were facing a very concrete distant learning scenario. In this context, our results showed again that students who work in a continuous way have the smallest chance of failure. However, in agreement with our previous findings, scores were not significantly higher. Again, the most relevant effect of continuous working was reducing the number of students who did not pass the course. The most important results here are related to the correlation between students failing and the ambient conditions created by the COVID-19 confinement. We have seen that students who got better results were those that were affected by the most drastic confinement (in 2020 spring), where they were not allowed to even go out of their houses from March to May. In this year, we have seen that students worked continuously and even got a higher effort in the middle of the course than at the very end. This correlates with the fact that restrictions were much lower in May than in March. In 2020/2021, restrictions also applied, but they were much lighter, allowing students to do many activities outside home (without reaching anyway the same level as before COVID-19 pandemic indeed). Our results show that students had in this year a similar pattern as those from courses before the pandemic. Results related to failure are lower to those from years before the pandemic and higher thatn 2019/2020. In conclusion, our results show a correlation between students' performance and the restrictions due to the pandemic (more restrictions implies better performance). This is a very interesting conclusion because we are demonstrating that distant learning is not a better strategy by itself (not worse either). We are showing that the lack of opportunities of doing other fun activities ended in a better performance by applying a more continuous learning strategy. In the future, where COVID-19 pandemic vanishes, it will be very important to find different strategies to increase students' motivation. Since the disappearance of other activities increased students' success, it is clear that learning should become an attractive activity by itself.

\section{Conclusions}

In this article we have demonstrated that the use of computers in education, especially in distant learning, is a very valuable tool both for learning and for analyzing results from the assessment process in the COVID-19 confinement. In this unprecedented scenario, we have been able to determine the elements that helped students and the influence of the time management in their autonomous learning. We have demonstrated that continuous working is not the only way to get good scores in higher education. However, it is a way of working that drastically reduces the risk of failing or dropping the subject. These conclusions were obtained after a rigorous data analysis in the first part of this study involving a set of 44 students from 
Early Childhood Education subjects and was confirmed in an additional study from Applied Computing subject including three different scenarios that included COVID-19 confinement.

We have presented a computer-assisted approach aimed to improve the self-regulated learning process of students by using adaptive tests. This method strongly increases the engagement of students and give us the opportunity of measuring accurately their autonomous learning activity.

By applying a numerical model that includes some information about the dynamics of the learning process, we have demonstrated that students' pattern of access is a relevant parameter to predict students' success. We have been able to identify two kinds of students as a function of their learning strategies: the ones that started to study earlier and more dispersed throughout time and those that started to study later and do it more concentrate. We found a statistically significant difference between those groups. By our findings, studying earlier and more dispersed maximizes probabilities of not failing in the final exam. Another interesting result is that using a strategy that involves a last-minute great effort instead of a continuous learning strategy does not implies necessarily failure since we have found both low and high scores in this case.

These results were confirmed when analyzing students' learning strategies in COVID-19 pandemic. In this case, students worked in a more continuous way when they were forced to stay at home (in 2020). In this year, the ratio of students who did not pass or skipped the final exam decreased. In previous and following years, they studied in the last minute, having worse results in those parameters. This fact suggests that the lack of fun activities in 2020 due to the confinement helped in students' engagement. Since COVID-19 confinement is a temporal situation, additional motivation strategies to improve students' engagement should be developed in the future.

\section{Acknowledgments}

The authors want to thank Pablo Molins-Ruano for his valuable comments.

\section{Declarations}

\section{Funding}

The research leading to these results received funding from from the Fondo SuperaCOVID19 (Banco Santander, CRUE and CSIC) under the project named "Desarrollo de herramientas de análisis de la fiabilidad de la evaluación en la universidad debida al confinamiento por COVID-19” FiabEvaluaCOVID19

\section{Conflicts of interest/Competing interests}

The authors have no conflicts of interest to declare that are relevant to the content of this article

\section{Availability of data and material}

Data is available under request.

\section{Code availability}

e-valUAM is available to all users in www.evaluam.eu

\section{Ethics approval}

This work involves data that have been collected from students of the Universidad Autónoma de Madrid. These data have been collected in the following form: 
1)It does not involve minors. information.

2)It has been collected anonymously. Students have been identified by a numerical code, avoiding any personal

3)Students have been informed by the lecturers that some information about their activity could be anonymously collected for statistical porpoises. Authors did not receive any objection.

4)The tasks related to this study were completely voluntary and they did not alter in any form students' activities, classes or assessment process.

In these conditions, we do not need to apply for ethics approval from our university since no personal data, minors or potentially hazardous activities were involved in the study.

Teachers related to the study (coauthors of the present manuscript) were also responsible for the subjects involved and agreed with the study.

\section{Consent to participate}

All participants consent to participate when applicable.

\section{Consent for publication}

All participants and authors gave permission for publication when applicable.

\section{References}

1 Aristovnik A, Kerzic D, Ravselj D, Tomazevic N, Umek L. Impacts of the COVID-19 Pandemic on Life of Higher Education Students: A Global Perspective. Sustainability. 2020;12(20). doi:10.3390/su12208438.

2 García-Peñalvo FJ, García-Holgado A, Vázquez-Ingelmo A, Sánchez-Prieto JC. Planning, Communication and Active Methodologies: Online Assessment of the Software Engineering Subject during the COVID-19 Crisis. RIED. Revista Iberoamericana de Educación a Distancia, 2021;24(2).

3 Molins-Ruano P, Jurado F, Rodríguez P, Atrio Cerezo S, Gómez Moñivas, S. Influence of the students' learning strategy on the evaluation scores. In Third international conference on Technological Ecosystems for Enhancing Multiculturality, proceedings teem'15, 2015 .

4 Gonzalez T, De La Rubia M, Hincz KP, Comas-Lopez M, Subirats L, Fort S, et al. Influence of COVID-19 confinement on students' performance in higher education. PloS one. 2020;15(10):e0239490.

5 Warfvinge P, Löfgreen J, Andersson K, Roxå T, Åkerman C. The rapid transition from campus to online teaching - how are students' perception of learning experiences affected? European Journal of Engineering Education 2021;1-19.

6 Turnbull D, Chugh R, Luck J. Transitioning to E-Learning during the COVID-19 pandemic: How have Higher Education Institutions responded to the challenge? Educ Inf Technol 2021;26:6401-6419

7 Sari T, Nayır F. Challenges in Distance Education During the (Covid-19) Pandemic Period. Qualitative Research in Education, 2020;9(3):328-360.

8 Talsma K, Robertson K, Thomas C, Norris K. COVID-19 Beliefs, Self-Efficacy and Academic Performance in First-year University Students: Cohort Comparison and Mediation Analysis. Front. Psychol., 2021;12:643408. 
9 Baltà Salvador R, Olmedo Torre N, Peña M, \& Renta Davids AI. Academic and emotional effects of online learning during the COVID-19 pandemic on engineering students. Education and information technologies, 2021;1-28. Advance online publication. 10 Drašler V, Bertoncelj J, Korošec M, Žontar TP, Ulrih NP, Cigić B. Difference in the Attitude of Students and Employees of the University of Ljubljana towards Work from Home and Online Education: Lessons from COVID-19 Pandemic. Sustainability, 2021;13(9):5118.

11 Zhou J, Zhang Q. A Survey Study on U.S. College Students' Learning Experience in COVID-19. Education sciences, 2021;11:248.

12 Lee Y. Effect of uninterrupted time-on-task on students' success in Massive Open Online Courses (MOOCs). Computers in Human Behavior, 2018;174-180.

13 Maldonado-Mahauad J, Perez-Sanagustin M, Kizilcec RF, Morales N, Munoz-Gama J. Mining theory-based patterns from Big data: Identifying self-regulated learning strategies in Massive Open Online Courses. Computers in Human Behavior. 2018;80;179-196.

14 Chiu YC, Hsu HJ, Wu J, Yang DL. Predicting student performance in MOOCs using learning activity data. Journal of Information Science and Engineering, 2018;34(5):1223-1235.

15 Louw J, Muller J, Tredoux C. Time-on-task, technology and mathematics achievement. Evaluation and Program Planning, 2008;31:41-50.

16 Eriksson T, Adawi T, Stöhr C. "Time is the bottleneck": a qualitative study exploring why learners drop out of MOOCs. Journal of Computing in Higher Education, 2017;29:133-146.

17 Nawrot I, Doucet A. Building engagement for MOOC students: introducing support for time management on online learning platforms. WWW '14 Companion: Proceedings of the 23rd International Conference on World Wide Web, $2014 ; 1077-1082$. 18 Wagner P, Schober B, Spiel C. Time students spend working at home for school. Learning and Instruction, 2008;18:309-320. 19 Narciss S, Sosnovsky S, Schnaubert L, Andrès E, Eichelmann A, Goguadze G, et al. Exploring feedback and student characteristics relevant for personalizing feedback strategies. Computers \& Education. 2014;71;56-76.

20 Chapelle CA., Douglas D. Assessing Language through Computer Technology (Cambridge Language Assessment). Cambridge: Cambridge University Press 2006.

21 Ortigosa A, Paredes P, \& Rodriguez P. AH-questionnaire: An adaptive hierarchical questionnaire for learning styles. Computers \& Education, 2010;54(4):999-1005.

22 Molins-Ruano P, González-Sacristán C, Díez F, Rodríguez P, Gómez Moñivas S. An adaptive model for computer-assisted assessment in programming skills. The International journal of engineering education, 2015;31:764-770.

23 Klinkenberg S, Straatemeier M, Van Der Maas HL. Computer adaptive practice of Maths ability using a new item response model for on the fly ability and difficulty estimation. Computers \& Education, 2011;57(2):1813-1824.

24 McGlohen M, Chang HH. Combining computer adaptive testing technology with cognitively diagnostic assessment. Behavior research methods, 2008;40(3):808-821. 
25 Molins-Ruano P, Rodríguez P, Atrio Cerezo S, Gómez Moñivas S. Modelling experts' behavior with e-valUAM to measure computer science skills. Computers in Human Behavior, 2016;61:378-385.

26 Lehmann T, Hähnlein I, Ifenthaler D. Cognitive, metacognitive and motivational perspectives on preflection in self-regulated online learning. Computers in Human Behavior, 2014;32:313-323.

27 Martin AJ, Lazendic G. Computer-Adaptive Testing: Implications for Students' Achievement, Motivation, Engagement, and Subjective Test Experience. Journal of Educational Psychology. 2018;110(1);27-45. 